\title{
Ventilation-Perfusion Scintigraphy Is More Sensitive than Multidetector CTPA in Detecting Chronic Thromboembolic Pulmonary Disease as a Treatable Cause of Pulmonary Hypertension
}

\author{
Nina Tunariu ${ }^{1}$, Simon J.R. Gibbs ${ }^{2,3}$, Zarni Win ${ }^{4}$, Wendy Gin-Sing ${ }^{2}$, Alison Graham ${ }^{1}$, Philip Gishen ${ }^{1}$, and \\ Adil AL-Nahhas ${ }^{3,4}$ \\ ${ }^{1}$ Department of Radiology, Hammersmith Hospital, London, United Kingdom; ${ }^{2}$ Department of Cardiology, Hammersmith Hospital, \\ London, United Kingdom; ${ }^{3}$ Imperial College, London, United Kingdom; and ${ }^{4}$ Department of Nuclear Medicine, Hammersmith Hospital, \\ London, United Kingdom
}

Pulmonary hypertension $(\mathrm{PH})$ is a progressive disease with a poor prognosis. Identifying chronic thromboembolic pulmonary disease as a cause of $\mathrm{PH}$ has major clinical implications as these patients could be potentially offered a surgical cure. Ventilationperfusion $(\mathrm{V} / \mathrm{Q})$ scintigraphy has a high sensitivity to detect embolic disease but its value has been challenged with the emergence of multidetector CT pulmonary angiography (CTPA). We compared the value of V/Q scintigraphy with CTPA in detecting chronic thromboembolic pulmonary disease. Methods: We retrospectively reviewed the results of V/Q scintigraphy and CTPA performed on patients who had been referred to the Pulmonary Hypertension Service at Hammersmith Hospital between 2000 and 2005. A total of 227 patients ( 85 males, 142 females; age range, 18-81 y; mean age, 42 y) had all tests done at Hammersmith Hospital and were included in the study. Interpretation of scans was according to the modified PIOPED (Prospective Investigation of Pulmonary Embolism Diagnosis) criteria. CTPA was considered as suggestive of chronic thromboembolic pulmonary disease if it showed visualization of the thrombus or webs, recanalization, perfusion abnormalities, stenosis, or strictures. Standard pulmonary angiography was performed via femoral approach. In $90 \%$ of the cases, CTPA and V/Q scintigraphy were performed within $10 \mathrm{~d}$. Results: Seventy-eight patients (group A) had a final diagnosis of chronic thromboembolic pulmonary hypertension (CTEPH) and 149 (group B) had non-CTEPH etiology. Among group $A, V / Q$ scintigraphy was reported as high probability in 75 patients, intermediate probability in 1 patient, and low probability in 2 patients. CTPA was positive in 40 patients and negative in 38 patients. Among group B, V/Q scintigraphy was reported as low probability in 134, intermediate probability in 7 , and high probability in 8 patients. CTPA was negative in 148 patients and false-positive in 1 patient. Statistical analysis showed V/Q scintigraphy to have a sensitivity of $96 \%-$ $97.4 \%$ and a specificity of $90 \%-95 \%$. CTPA showed a sensitivity

Received Dec. 29, 2006; revision accepted Feb. 15, 2007.

For correspondence or reprints contact: Adil AL-Nahhas, MSc, Department of Nuclear Medicine, Hammersmith Hospital, Du Cane Rd., London, W12 OHS, U.K.

E-mail: aal-nahhas@hhnt.org

COPYRIGHT @ 2007 by the Society of Nuclear Medicine, Inc. of $51 \%$ and a specificity of $99 \%$. Conclusion: Our results demonstrate that V/Q scintigraphy has a higher sensitivity than CTPA in detecting CTEPH as a potential curable cause of $\mathrm{PH}$.

Key Words: chronic thromboembolic pulmonary hypertension; V/Q scintigraphy; multidetector CTPA

J Nucl Med 2007; 48:680-684

DOI: 10.2967/jnumed.106.039438

$\mathbf{P}$ ulmonary hypertension $(\mathrm{PH})$ is a progressive disease with a poor prognosis, defined as a mean pulmonary artery pressure $>25 \mathrm{~mm} \mathrm{Hg}$ (>30 $\mathrm{mm} \mathrm{Hg}$ during exertion) measured during right heart catheterization. It can present as an idiopathic condition or as a complication of various medical conditions, including chronic cardiac and pulmonary diseases (1-3).

An accurate diagnosis of the cause of pulmonary hypertension determines the management and prognosis. Patients with chronic thromboembolic pulmonary hypertension (CTEPH) represent a distinct category of PH as they can potentially be offered curable surgery in the form of pulmonary endarterectomy. This operative procedure has gained wide acceptance and achieved good success rates, with a reported mortality rate of $4 \%-8 \%$ (4-7).

Without treatment, the prognosis of CTEPH is very poor, with a quoted survival rate of $30 \%$ over a period of $5 \mathrm{y}$ for patients with mean pulmonary artery pressure $>30 \mathrm{~mm} \mathrm{Hg}$ $(8,9)$.

Recent literature have shown that up to $3.8 \%$ of patients diagnosed with acute pulmonary embolism (PE) develop CTEPH at 2-y follow-up (10) and suggest that the incidence of CTEPH is much higher than previously thought $(10,11)$. Furthermore, up to $63 \%$ of patients diagnosed with CTEPH may not have a previous history of documented PE (12).

Identifying CTEPH as the cause of $\mathrm{PH}$ is facilitated by several imaging techniques, including ventilation-perfusion 
(V/Q) scintigraphy, multidetector CT pulmonary angiography (CTPA), high-resolution CT (HRCT), pulmonary digital subtraction angiography (DSA), and, more recently, magnetic resonance angiography $(13,14)$.

The introduction of spiral CTPA has challenged the role of V/Q scanning in diagnosing acute PE (15). In the United Kingdom, it has become the first line of the investigation in patients with suspected acute PE (16).

Further advances in CT technology, with the introduction of multidetector CTPA and multiplanar reconstruction have further improved the sensitivity and specificity of this technique with small studies quoting values of $96 \%$ and $98 \%$, respectively (17). However, a recent large multicenter study has shown these figures to be lower, with a sensitivity of $83 \%$ and a specificity of $96 \%$ for CTPA, with even a lower sensitivity for segmental $(68 \%)$ and subsegmental $(25 \%)$ branches (18).

This has been adopted by PIOPED (Prospective Investigation of Pulmonary Embolism Diagnosis) II investigators and is reflected in the new guidelines published in January 2007, which have approved multidetector CTPA as the firstline imaging procedure in most patients suspected of having PE (19).

However, the sensitivity of multidetector CTPA in detecting chronic thromboembolic pulmonary disease remains variable. There are few publications in this respect, including a study published by Pitton et al. showing multidetector CTPA to have a sensitivity of $70.4 \%$ for segmental and $63.6 \%$ for subsegmental branches when compared with pulmonary DSA (20).

On the other hand, several studies have shown that highprobability and intermediate-probability V/Q scintigraphy has a high sensitivity and specificity for distinguishing patients with chronic pulmonary thromboembolic disease from other causes of $\mathrm{PH}$, to the extent that normal V/Q scintigraphy can comfortably rule out CTEPH (21-27). To our knowledge, there is no published study that compares the value of V/Q scintigraphy with that of multidetector CTPA in the diagnosis of CTEPH. Our aim was to perform a head-tohead comparison of the 2 techniques.

\section{MATERIALS AND METHODS}

We retrospectively reviewed the results of V/Q scintigraphy and CTPA performed on 500 patients with $\mathrm{PH}$ referred to Hammersmith Hospital, London, between 2000 and 2005. Patients selected for inclusion were those who had all of their imaging tests performed at Hammersmith Hospital rather than in the referring hospitals. Of the 500 patients, 227 (85 males, 142 females; age range, 18-81 y; mean age, 42 y) fulfilled these criteria. For such retrospective review neither ethical approval nor informed consent was required. The imaging reports have been retrieved from the Hammersmith Hospital picture archiving and communication system (PACS) archive. The final diagnosis of CTEPH or nonCTEPH was obtained from the Hammersmith Hospital Pulmonary Hypertension Service case records. The information obtained from medical history, laboratory tests, and other investigationssuch as echocardiography, CTPA, V/Q, right heart cardiac cath- eterization, and pulmonary angiography—was used to obtain a final diagnosis.

The identification of the PH clinical class (e.g., CTEPH vs. non-CTEPH etiology) was made in all patients in accordance with the guidelines of the European Society of Cardiology and the American College of Chest Physicians (1-3).

\section{V/Q Scintigraphy}

V/Q scintigraphy was performed using ${ }^{99 \mathrm{~m}} \mathrm{Tc}$-labeled macroaggregated albumin $\left({ }^{99 \mathrm{~m}} \mathrm{Tc}-\mathrm{MAA}\right)$ and ${ }^{81 \mathrm{~m}} \mathrm{Kr}$ gas $\left({ }^{81} \mathrm{Kr}\right)$. Images were acquired using an ECAM dual-head $\gamma$-camera (Siemens Medical Solutions USA, Inc.). The perfusion images were acquired after intravenous administration of $100 \mathrm{MBq}$ of ${ }^{99 \mathrm{~m}} \mathrm{Tc}-$ MAA with the patient in the supine position. The images were acquired in 4 standard projections (anterior, posterior, right posterior oblique, and left posterior oblique) with a medium-energy, general-purpose collimator. An energy window of $20 \%$ centered on the energy peak of ${ }^{99 \mathrm{~m}} \mathrm{Tc}(140 \mathrm{keV})$, and a matrix of $128 \times 128$ pixels was used and approximately 400,000 counts accumulated per view. Ventilation images using ${ }^{81 \mathrm{~m}} \mathrm{Kr}$ were acquired immediately after each perfusion view. ${ }^{81 \mathrm{~m}} \mathrm{Kr}$ was inhaled through a mouthpiece. The acquisition parameters were the same as those used for the perfusion study, but the $20 \%$ window was centered on the energy peak of ${ }^{81 \mathrm{~m}} \mathrm{Kr}(190 \mathrm{keV})$. Images were reported from a Hermes workstation (Nuclear Diagnostics) by experienced nuclear medicine physicians. Images were interpreted according to the modified PIOPED criteria $(28,29)$. A high-probability scan was suggestive of chronic thromboembolic pulmonary disease, whereas a low-probability scan was suggestive of nonchronic thromboembolic pulmonary disease etiology. For intermediate-probability scans, 2 separate datasets were produced and analyzed. In one dataset, intermediate-probability scans were considered as suggestive of chronic thromboembolic pulmonary disease and, in the other dataset, the intermediate-probability scans were considered as not suggestive of chronic thromboembolic pulmonary disease. This was in accordance with previous similar studies (22).

\section{Multidetector CTPA}

CTPA was acquired with multidetector row CT scanners. The first 24 studies were acquired with a Somaton Four Plus CT scanner (Siemens Medical Solutions) using $4 \times 3 \mathrm{~mm}$ collimation and 2.5-mm reconstruction. From March 2002, the remaining 203 studies were acquired on a Light Speed CT scanner (GE Healthcare), using $8 \times 1.25 \mathrm{~mm}$ collimation with $1.25-\mathrm{mm}$ reconstruction. A bolus injection of $100 \mathrm{~mL}$ of nonionic iodinated contrast medium $(300 \mathrm{mg} / \mathrm{mL})$ was administered intravenously via a peripheral cannula at $4 \mathrm{~mL} / \mathrm{s}$ via a power injector with a variablestart delay. This was determined using automatic bolus trigger software with a circular region of interest positioned at the level of main pulmonary trunk. The images were acquired in the craniocaudal direction with the $z$-axis coverage and the field of view chosen to include the entire thorax, from the apex to the base of the lungs.

All CTPA was reported by experienced radiologists using PACS workstations (GE Healthcare) as well as Siemens and General Electric workstations to assess multiplanar reconstructions. A report was considered as suggestive of chronic thromboembolic pulmonary disease if it stated visualization of the thrombus, calcified thrombus, recanalization, sudden change in vessel caliber, strictures, poststenotic dilatation, webs, or perfusion abnormality (30-34). The presence of mosaic perfusion, defined as areas of 
increased and decreased attenuation and vascularity without evidence of destruction or displacement of pulmonary vessels, was also noted. However, its presence alone in the absence of other signs was not considered as diagnostic of chronic thromboembolic pulmonary disease (35-37).

\section{Pulmonary DSA}

The indications for referring patients for pulmonary DSA included (a) clinical suspicion of CTEPH, (b) at least one positive imaging modality for chronic thromboembolic pulmonary disease, and (c) whether the patient was being assessed for pulmonary thrombendarterectomy. Pulmonary DSA was performed in 61 patients by experienced vascular radiologists via a femoral venous approach using a 7-French Grollman pigtail catheter (William Cook Europe) placed in the main pulmonary trunk. More selective studies of the intrapulmonary artery branches were performed in some patients with a 7-French headhunter catheter (Cordis Europa). Images were obtained with an HDI 5000 fluoroscopy unit (Philips) after administration of $20 \mathrm{~mL}$ of nonionic iodinated contrast medium $(300 \mathrm{mg} / \mathrm{mL})$, per DSA run, at $7 \mathrm{~mL} / \mathrm{s}$ via a pump injector or by hand injection with a maximum volume limit of $300 \mathrm{~mL}$. The minimum projections per hemithorax included frontal and posterior oblique projections, with extra views obtained as required.

\section{RESULTS}

All 227 patients included in this analysis had both V/Q scintigraphy and CTPA performed at Hammersmith Hospital, with most (90\%) of the CTPA being performed within $10 \mathrm{~d}$ from the V/Q scintigraphy and $73 \%$ being performed within $48 \mathrm{~h}$. When chronic thromboembolic pulmonary disease was highly suspected, pulmonary DSA was performed in patients who were clinically fit to undergo pulmonary endarterectomy.

In patients with CTEPH (group $\mathrm{A}, n=78$ patients), the final diagnosis was confirmed by pulmonary DSA in 61 patients. In the remaining 17 patients, there was strong clinical and imaging suspicion of chronic thromboembolic pulmonary disease. However, these patients did not consent to surgery or were considered unfit for surgery and, therefore, not referred for pulmonary DSA.

In patients with non-CTEPH (group B, $n=149$ patients), the clinical and imaging data did not suggest thromboembolic etiology, so there was no clinical indication to refer them for pulmonary DSA. The final diagnosis of PH of nonthromboembolic etiology was achieved by a consensus based on clinical and imaging data discussed at a multidisciplinary meeting.

\section{Patients with CTEPH: Group A}

CTPA was reported as showing features suggestive of chronic thromboembolic pulmonary disease in 40 of 78 patients but was falsely negative in 38 of 78 cases, giving a sensitivity of $51 \%$. The V/Q scintigraphy showed high probability in 75 patients, intermediate probability in 1 patient, and low probability in 2 patients. When the intermediateprobability scans were considered suggestive of chronic thromboembolic pulmonary disease (grouped with the high probability), the V/Q sensitivity was $97.4 \%$, and when intermediate probability was considered as negative for chronic thromboembolic pulmonary disease (grouped with low probability), the sensitivity was $96 \%$ (Tables 1 and 2).

\section{Patients with Non-CTEPH: Group B}

CTPA did not show features suggestive of chronic thromboembolic pulmonary disease in 148 of 149 patients with one false-positive case. V/Q scintigraphy showed low probability in 134 patients, high probability in 8 patients, and intermediate in 7 patients.

The specificity of CTPA was $99 \%$. The V/Q specificity was $90 \%$ when the intermediate-probability scans were analyzed as suggestive of chronic thromboembolic pulmonary disease and $95 \%$ when the intermediate scans were considered negative for chronic thromboembolic pulmonary disease (Tables 1 and 2).

\section{Mosaic Perfusion}

Mosaic perfusion was reported in 53 of the total 227 CTPA scans (23.34\%). Among those, 43 (18.94\%) were in group A and 10 (4.41\%) were in group B.

Thus, the CTPA showed evidence of mosaic perfusion in $55 \%$ (43/78) of patients with CTEPH (group A).

\section{DISCUSSION}

The sensitivity and specificity of the V/Q scintigraphy in diagnosing chronic thromboembolic pulmonary disease shown in our study are similar to those of a smaller group (75 patients with PH) studied by Worsley at al. (22). They found that the combination of high- and indeterminateprobability V/Q scintigraphy had a sensitivity of $100 \%$ for detecting patients with CTEPH; however, the specificity was $86 \%$ (22). Other studies have also highlighted the value of V/Q scintigraphy in the diagnosis of CTEPH (21-27).

In our study, we approach the V/Q scintigraphy analysis from 2 perspectives. First (V/Q (1)), we considered both the high- and intermediate-scans as suggestive of chronic pulmonary thromboembolic disease, which resulted in a sensitivity of $97.4 \%$, specificity of $90 \%$, accuracy of $92.5 \%$, negative predictive value (NPV) of $98.5 \%$, and positive predictive value (PPV) 83.5\%, respectively (Table 2).

When we analyzed the data considering just the highprobability scans as suggestive of chronic pulmonary thromboembolic disease (V/Q (2)), the sensitivity was 96.2\%, whereas the specificity increased to $94.6 \%$. The overall accuracy was $95.2 \%$ with a NPV of $97.9 \%$ and a PPV of 90.3\% (Table 2).

TABLE 1

Summary of V/Q Scans and CTPA Results

\begin{tabular}{lrrrrrr}
\hline & \multicolumn{3}{c}{ V/Q } & & \multicolumn{2}{c}{ CTPA } \\
\cline { 2 - 5 } \cline { 5 - 6 } Group & Low & Intermediate & High & & \\
\hline A $(n=78)$ & 2 & 1 & 75 & 38 & 40 \\
B $(n=149)$ & 134 & 7 & 8 & 148 & 1 \\
\hline
\end{tabular}


TABLE 2

Summary of Performance Indicators for V/Q Scintigraphy and CTPA

\begin{tabular}{llcc}
\hline & \multicolumn{2}{c}{ Scintigraphy } & \\
\cline { 2 - 3 } Indicator & V/Q (1) & V/Q (2) & CTPA \\
\hline Sensitivity (\%) & 97.4 & 96.2 & 51.3 \\
Specificity (\%) & 90 & 94.6 & 99.3 \\
Accuracy (\%) & 92.5 & 95.2 & 82.8 \\
NPV (\%) & 98.5 & 97.9 & 79.7 \\
PPV (\%) & 83.5 & 90.3 & 97.6
\end{tabular}

*Intermediate with high-probability scans as indicative of CTEPH. ${ }^{\dagger}$ Only high-probability scans as indicative of CTEPH.

$\mathrm{NPV}=$ negative predictive value; $\mathrm{PPV}=$ positive predictive value.

The sensitivity of CTPA in our study was significantly lower compared with V/Q scintigraphy in detecting chronic thromboembolic pulmonary disease changes. However, it was similar with that reported by Pitton et al., who had analyzed 994 vessel segments in 14 patients with CTEPH and had both CTPA and selective pulmonary DSA (20). Their analysis showed CTPA to be significantly inferior to selective DSA, with a $67 \%$ overall concordance in diagnosing CTEPH. Furthermore, the nonocclusive changes of the vessels were significantly underdiagnosed by CT, with a concordance between CT and DSA of 23.1\% (20).

\section{False-Negative V/Q Scintigraphy}

The 2 patients from group A who had their V/Q scintigraphy interpreted as low probability did not have the diagnosis of CTEPH confirmed on pulmonary DSA. The final diagnosis was made by CTPA, which showed calcified thrombus in one patient and narrowed but patent pulmonary arterial branches in the other patient. By corroborating the patient clinical history and the CT findings, it was concluded that these patients most likely had previous PE and the pulmonary vessels had recanalized.

V/Q scintigraphy that was interpreted as intermediate probability was confirmed on pulmonary DSA to be CTEPH. The CT was reported as showing severe emphysematous changes but failed to show signs of CTEPH. It was the V/Q result together with the clinical history that prompted the clinicians to request pulmonary DSA.

\section{False-Positive V/Q Scintigraphy}

Eight V/Q scans were reported as high probability and 7 were reported as intermediate probability among the nonCTEPH group. None of these patients had pulmonary DSA. The clinical diagnoses were idiopathic pulmonary arterial hypertension, pulmonary arterial hypertension associated with atrial septal defect, pulmonary venoocclusive disease, and PH related to parenchymal lung disease, such as emphysema or pulmonary fibrosis (Table 3 ).
TABLE 3

Summary of False-Positive V/Q Scans

\begin{tabular}{cl}
\hline V/Q & \multicolumn{1}{c}{ Diagnosis } \\
\hline Intermediate & IPAH $(n=4)$ \\
probability $(n=7)$ & IPAH and emphysema $(n=1)$ \\
& ASD and pulmonary fibrosis $(n=1)$ \\
& Pulmonary fibrosis $(n=1)$ \\
High probability & IPAH $(n=3)$ \\
$(n=8)$ & PVOD $(n=1)$ \\
& APAH and ASD $(n=2)$ \\
& Scleroderma and pulmonary fibrosis \\
& $(n=1)$ \\
& Apical bullae: apical mismatch on \\
& V/Q $(n=1)$
\end{tabular}

IPAH = idiopathic pulmonary arterial hypertension; ASD = atrial septal defect; PVOD = pulmonary venoocclusive disease; APAH = associated pulmonary arterial hypertension.

\section{Mosaic Attenuation Pattern}

The combination of a mosaic pattern and disparity in the size of segmental vessels on HRCT was found in a study by Bergin et al. to reliably distinguish patients with CPTEH (33). Similarly, Schwickert et al. analyzed the CT findings in 75 patients diagnosed with CTEPH and found mosaic perfusion in $77 \%$ (34). However, the mosaic perfusion pattern can also be found in patients with $\mathrm{PH}$ due to other causes (31-37), and up to $12 \%$ of patients with idiopathic pulmonary arterial hypertension will have mosaic perfusion on CTPA (35). In our study, mosaic perfusion was present in only $55 \%$ of the CTPA performed in group A patients and $20 \%$ of group B patients. This confirms that, although the mosaic pattern is more common in CTEPH, it is not a specific feature.

\section{CONCLUSION}

Our findings confirm that normal V/Q scintigraphy practically rules out the presence of CTEPH, whereas normal CTPA does not exclude the presence of CTEPH. In contrast, the presence of high probability on V/Q scintigraphy makes CTEPH the most likely diagnosis, although other conditions-including pulmonary venoocclusive disease-may result in similar findings. Our results suggest that grouping intermediate-probability with low-probability V/Q scans as indicative of non-CTEPH etiology would maintain a high sensitivity and improve specificity, NPV, and PPV. We have shown that V/Q scintigraphy, which is widely available and easy to perform, has a higher sensitivity than multidetector CTPA as well as very good specificity in detecting chronic pulmonary thromboembolic disease as a potential curable cause of $\mathrm{PH}$.

\section{REFERENCES}

1. Galie N, Torbicki A, Barst R, et al. Guidelines on diagnosis and treatment of pulmonary arterial hypertension: the Task Force on Diagnosis and Treatment of 
Pulmonary Arterial Hypertension of the European Society of Cardiology. Eur Heart J. 2004;25:2243-2278.

2. Simonneau G, Galie N, Rubin LJ, et al. Clinical classification of pulmonary hypertension. J Am Coll Cardiol. 2004;43(suppl):5S-12S.

3. McGoon M, Gutterman D, Steen V, et al. Screening, early detection, and diagnosis of pulmonary arterial hypertension: ACCP evidence-based clinical practice guidelines. Chest. 2004;126(suppl):14S-34S.

4. Fedullo PF, Auger WR, Kerr KM, Kim NH. Chronic thromboembolic pulmonary hypertension. Semin Respir Crit Care Med. 2003;24:273-286.

5. Jamieson SW, Kapelanski DP, Sakakibara N, et al. Pulmonary endarterectomy: experience and lessons learned in 1,500 cases. Ann Thorac Surg. 2003;76:14571462.

6. Kim NH. Assessment of operability in chronic thromboembolic pulmonary hypertension. Proc Am Thorac Soc. 2006;3:584-588.

7. Doyle RL, McCrory D, Channick RN, Simonneau G, Conte J. American College of Chest Physicians: surgical treatments/interventions for pulmonary arterial hypertension-ACCP evidence-based clinical practice guidelines. Chest. 2004; 126(suppl):63S-71S.

8. Lewczuk J, Piszko P, Jagas J, et al. Prognostic factors in medically treated patients with chronic pulmonary embolism. Chest. 2001;119:818-823.

9. Riedel M, Stanek V, Widimsky J, Prerovsky I. Long-term follow-up of patients with pulmonary thromboembolism: late prognosis and evolution of hemodynamic and respiratory data. Chest. 1982;81:151-158.

10. Pengo V, Lending AW, Prins MH, et al. Thromboembolic Pulmonary Hypertension Study Group: incidence of chronic thromboembolic pulmonary hypertension after pulmonary embolism. N Engl J Med. 2004;350:2257-2264.

11. Becattini C, Agnelli G, Pesavento R, et al. Incidence of chronic thromboembolic pulmonary hypertension after a first episode of pulmonary embolism. Chest. 2006;130:172-175.

12. Lang IM. Chronic thromboembolic pulmonary hypertension: not so rare after all. N Engl J Med. 2004;350:2236-2238.

13. Hoeper MM, Mayer E, Simonneau G, Rubin LJ. Chronic thromboembolic pulmonary hypertension. Circulation. 2006;113:2011-2020.

14. Dartevelle P, Fadel E, Mussot S, et al. Chronic thromboembolic pulmonary hypertension. Eur Respir J. 2004;23:637-648.

15. Hayashino Y, Goto M, Noguchi Y, Fukui T. Ventilation-perfusion scanning and helical CT in suspected pulmonary embolism: meta-analysis of diagnostic performance. Radiology. 2005;234:740-748.

16. British Thoracic Society guidelines for the management of suspected acute pulmonary embolism: British Thoracic Society Standards of Care Committee Pulmonary Embolism Guideline Development Group. Thorax. 2003;58:470483.

17. Coche E, Verschuren F, Keyeux A, et al. Diagnosis of acute pulmonary embolism in outpatients: comparison of thin-collimation multi-detector row spiral CT and planar ventilation-perfusion scintigraphy. Radiology. 2003;229:757-765.

18. Stein PD, Fowler SE, Goodman LR, et al. Multidetector computed tomography for acute pulmonary embolism. N Engl J Med. 2006;354:2317-2327.

19. Stein PD, Woodard PK, Weg JG, et al. PIOPED II Investigators: diagnostic pathways in acute pulmonary embolism-recommendations of the PIOPED II Investigators. Radiology. 2007;242:15-21.
20. Pitton MB, Kemmerich G, Herber S, Schweden F, Mayer E, Thelen M. Chronic thromboembolic pulmonary hypertension: diagnostic impact of multislice-CT and selective pulmonary-DSA. Rofo. 2002;174:474-479.

21. Bergin CJ, Hauschildt J, Rios G, Belezzuoli EV, Huynh T, Channick RN. Accuracy of MR angiography compared with radionuclide scanning in identifying the cause of pulmonary arterial hypertension. AJR. 1997;168:1549-1555.

22. Worsley DF, Palevsky HI, Alavi A. Ventilation-perfusion lung scanning in the evaluation of pulmonary hypertension. J Nucl Med. 1994;35:793-796.

23. Chapman PJ, Bateman ED, Benatar SR. Primary pulmonary hypertension and thromboembolic pulmonary hypertension: similarities and differences. Respir Med. 1990;84:485-488.

24. Moser KM, Page GT, Ashburn WL, Fedullo PF. Perfusion lung scans provide a guide to which patients with apparent primary pulmonary hypertension merit angiography. West J Med. 1988;148:167-170.

25. Lisbona R, Kreisman H, Novales-Diaz J, Derbekyan V. Perfusion lung scanning: differentiation of primary from thromboembolic pulmonary hypertension. AJR. 1985;144:27-30.

26. D'Alonzo GE, Bower JS, Dantzker DR. Differentiation of patients with primary and thromboembolic pulmonary hypertension. Chest. 1984;85:457-461.

27. Fishman AJ, Moser KM, Fedullo PF. Perfusion lung scans vs pulmonary angiography in evaluation of suspected primary pulmonary hypertension. Chest. 1983;84:679-683.

28. Freitas JE, Sarosi MG, Nagle CC, Yeomans ME, Freitas AE, Juni JE. Modified PIOPED criteria used in clinical practice. J Nucl Med. 1995;36:1573-1578.

29. Freeman LM, Krynyckyi BR, Zuckier LS. Enhanced lung scan diagnosis of pulmonary embolism with the use of ancillary scintigraphic findings and clinical correlation. Semin Nucl Med. 2001;31:143-157.

30. Remy-Jardin M, Duhamel A, Deken V, Bouaziz N, Dumont P, Remy J. Systemic collateral supply in patients with chronic thromboembolic and primary pulmonary hypertension: assessment with multi-detector row helical CT angiography. Radiology. 2005;235:274-281.

31. King MA, Ysrael M, Bergin CJ. Chronic thromboembolic pulmonary hypertension: CT findings. AJR. 1998;170:955-960.

32. King MA, Bergin CJ, Yeung DW, et al. Chronic pulmonary thromboembolism: detection of regional hypoperfusion with CT. Radiology. 1994;191:359-363.

33. Bergin CJ, Rios G, King MA, Belezzuoli E, Luna J, Auger WR. Accuracy of high-resolution $\mathrm{CT}$ in identifying chronic pulmonary thromboembolic disease. AJR. 1996;166:1371-1377.

34. Schwickert HC, Schweden F, Schild HH, et al. Pulmonary arteries and lung parenchyma in chronic pulmonary embolism: preoperative and postoperative CT findings. Radiology. 1994;191:351-357.

35. Sherrick AD, Swensen SJ, Hartman TE. Mosaic pattern of lung attenuation on CT scans: frequency among patients with pulmonary artery hypertension of different causes. AJR. 1997;169:79-82.

36. Worthy SA, Muller NL, Hartman TE, Swensen SJ, Padley SP, Hansell DM. Mosaic attenuation pattern on thin-section CT scans of the lung: differentiation among infiltrative lung, airway, and vascular diseases as a cause. Radiology. 1997;205:465-470.

37. Stern EJ, Swensen SJ, Hartman TE, Frank MS. CT mosaic pattern of lung attenuation: distinguishing different causes. AJR. 1995;165:813-816. 